Supporting Information

\title{
Fluorine-Free Superhydrophobic Surface by Single-Step-Synthesized \\ Homogeneous Polymeric Raspberry Nanoparticle Coating
}

$$
\text { Gunwoo Kim }{ }^{1,2, *} \text {, Kyuin Park² and Sungho Jin²,3 }
$$

1. Materials Science and Engineering, University of California, San Diego, 9500 Gilman

$$
\text { Dr., La Jolla, California 92093, United States }
$$

2. NanoSD, Inc., 11575 Sorrento Valley Rd., Suite 211, San Diego, California, 92121, United States

3. Department of Mechanical \& Aerospace Engineering, University of California, San

Diego, 9500 Gilman Dr., La Jolla, California 92093, United States

*Corresponding author. Email: creaventure@gmail.com (G.K.)

Number of pages: 7

Number of table: 1

Number of figures: 4 
This PDF includes following figures.

Supplementary text

Table S1. Surface tension components of materials and a contact angle with water.

Figure S1. Size distributions of raspberry nanoparticles.

Figure S2. FT-IR spectra of raspberry polymer nanoparticles.

Figure S3. SEM image for raspberry nanoparticles synthesized with trimethyl benzene as a sacrificial solvent.

Figure S4. Contact angle of water droplet on PS raspberry nanoparticle coated on the surface in response to the density of nanoparticles on the substrate. 
Supplementary text

Hansen solubility parameters of xylene and trimethyl benzene

Hansen solubility is utilized to evaluate a solubility between two materials. Hansen solubility components which is composed of dispersion forces $\left(\delta_{d}\right)$, dipolar intermolecular forces $\left(\delta_{p}\right)$ and hydrogen bonds $\left(\delta_{h}\right)$. The Hansen solubility components of xylene is $\delta_{d}=18.411, \delta_{p}=0.892$ and $\delta_{h}=0$ while that of xylene is $\delta_{d}=19.606, \delta_{p}=0.802$ and $\delta_{h}=0$. Two materials values are similar since they have analogous molecular structures. This indicates trimethyl benzene could be an alternative sacrificial solvent replacing xylene to synthesize raspberry polymeric nanoparticles. 
Table S1. Surface tension components of materials and a contact angle (CA) with water

\begin{tabular}{|c|c|c|c|c|c|c|c|}
\hline & $\begin{array}{c}\gamma \\
\left(\mathrm{mJ} / \mathrm{m}^{2}\right)\end{array}$ & $\begin{array}{c}\gamma^{L W} \\
\left(\mathrm{~mJ} / \mathrm{m}^{2}\right)\end{array}$ & $\begin{array}{c}\gamma^{A B} \\
\left(\mathrm{~mJ} / \mathrm{m}^{2}\right)\end{array}$ & $\begin{array}{c}\gamma^{+} \\
\left(\mathrm{mJ} / \mathrm{m}^{2}\right)\end{array}$ & $\begin{array}{c}\gamma^{-} \\
\left(\mathrm{mJ} / \mathrm{m}^{2}\right)\end{array}$ & $\mathrm{CA}\left({ }^{\circ}\right)$ & Ref. \\
\hline Water & 72.8 & 21.8 & 52 & 25.5 & 25.5 & 0 & - \\
\hline $\mathrm{PEO}$ & 45.9 & 45.9 & 0 & 0 & 66 & 5.08 & $(1)$ \\
\hline $\mathrm{SiO}_{2}$ & 59 & 43.72 & 15.29 & 1.74 & 33.53 & 33.44 & $(2)$ \\
\hline STS & 40.4 & 29.4 & 11 & 0.863 & 35.27 & 49.59 & (3) \\
\hline Polyvinyl alcohol & 42 & 42 & 0 & 0 & 32 & 51.97 & (1) \\
\hline PVP & 43.4 & 43.4 & 0 & 0 & 29.7 & 53.05 & $(4)$ \\
\hline Polyvinyl acetate & 44.5 & 42.6 & 1.9 & 0.04 & 22.3 & 58.66 & (6) \\
\hline Cellulose Acetate & 38 & 38 & 0 & 0 & 25 & 61.03 & $(6)$ \\
\hline Nylon 6,6 & 37.7 & 36.4 & 1.3 & 0.02 & 21.6 & 64.01 & $(5)$ \\
\hline PMMA & 40 & 40 & 0 & 0 & 14.6 & 70.04 & (7) \\
\hline Cellulose nitrate & 45.2 & 44.7 & 0.5 & 0.01 & 10.7 & 71.02 & $(6)$ \\
\hline Polyimide & 44 & 42.5 & 1.5 & 0.1 & 6 & 77.30 & $(8)$ \\
\hline PVC & 43 & 40.2 & 2.8 & 0.4 & 5.1 & 77.62 & (6) \\
\hline Polyvinyl fluoride & 36.8 & 34.1 & 2.7 & 8.3 & 0.2 & 77.83 & (9) \\
\hline PET & 45.9 & 44.6 & 1.3 & 0.1 & 4.4 & 78.96 & $(8)$ \\
\hline Polyurethanes & 30 & 30.9 & -0.9 & 0.02 & 10 & 80.13 & (10) \\
\hline $\mathrm{ABS}$ & 42 & 39.4 & 2.6 & 1 & 1.7 & 82.83 & $(6)$ \\
\hline Ethyl cellulose & 28.6 & 27.4 & 1.2 & 0.04 & 8.6 & 83.91 & $(11)$ \\
\hline PBT & 47.1 & 45.8 & 1.3 & 0.8 & 0.6 & 84.28 & $(12)$ \\
\hline PVDF & 40.3 & 40.4 & -0.1 & 0.002 & 2.6 & 87.41 & (9) \\
\hline $\mathrm{ZrO}_{2}$ & 41.21 & 40.97 & 0.24 & 1.6 & 0.1 & 87.69 & $(2)$ \\
\hline PS & 42 & 42 & 0 & 0 & 1.1 & 91.33 & $(5)$ \\
\hline Polytrifluoroethylene & 25.4 & 23.1 & 2.3 & 1.8 & 0.7 & 94.66 & $(9)$ \\
\hline $\mathrm{La}_{2} \mathrm{O}_{3}$ & 40.87 & 40.7 & 0.17 & 0.22 & 0.03 & 95.31 & $(2)$ \\
\hline $\mathrm{Nd}_{2} \mathrm{O}_{3}$ & 0.76 & 32.17 & 0.58 & 0.99 & 0.09 & 95.33 & (2) \\
\hline PTFE & 21.5 & 19.6 & 1.9 & 0.3 & 3.2 & 96.20 & (13) \\
\hline Polyethylene & 28.1 & 27.4 & 0.7 & 0.1 & 1.2 & 97.63 & $(8)$ \\
\hline $\mathrm{Y}_{2} \mathrm{O}_{3}$ & 27.2 & 26.83 & 0.37 & 0.22 & 0.15 & 102.52 & $(2)$ \\
\hline PDMS & 19.31 & 18.4 & 0.91 & 0.32 & 0.64 & 105.09 & (14) \\
\hline $\mathrm{Gd}_{2} \mathrm{O}_{3}$ & 24.5 & 24.4 & 0.1 & 0.058 & 0.069 & 107.25 & (3) \\
\hline $\mathrm{Er}_{2} \mathrm{O}_{3}$ & 24 & 23.9 & 0.1 & 0.056 & 0.07 & 107.66 & (3) \\
\hline $\mathrm{CeO}_{2}$ & 23.7 & 23.7 & 0 & 0.035 & 0 & 110.46 & (3) \\
\hline $\mathrm{PP}$ & 25.7 & 25.7 & 0 & 0 & 0 & 110.47 & (7) \\
\hline Polyisobutylene & 25 & 25 & 0 & 0 & 0 & 111.02 & (7) \\
\hline FEP & 17.9 & 17.9 & 0 & 0 & 0 & 117.21 & $(7)$ \\
\hline
\end{tabular}




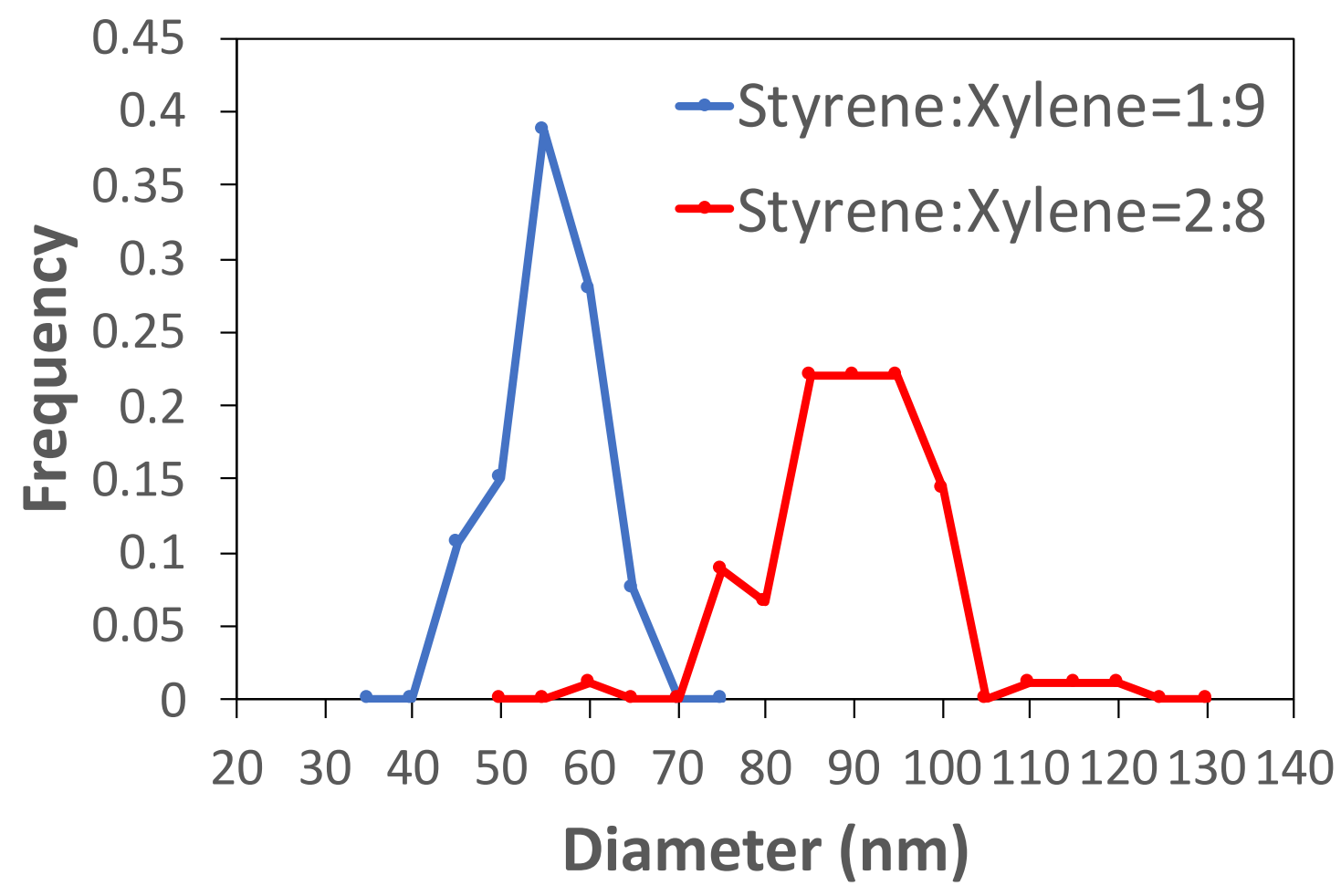

Figure S1. Size distributions of raspberry nanoparticles prepared by the feeding ratio of styrene : xylene $=1: 9$ (blue line) and $2: 8$ (red line).

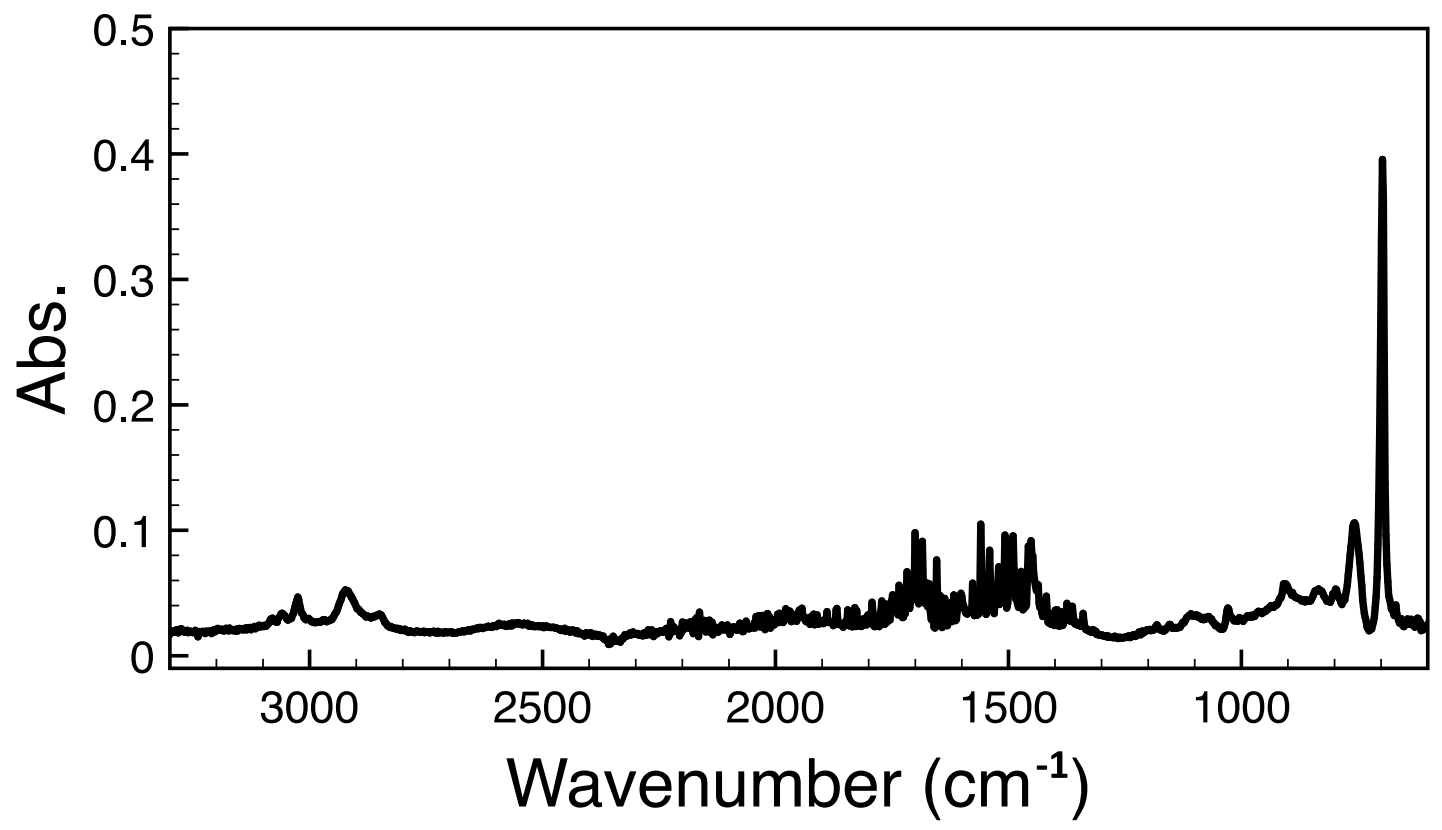

Figure S2. FT-IR spectra of raspberry polymer nanoparticles. The strong peak of $1200 \mathrm{~cm}^{-1}$ comes from pure PS. 


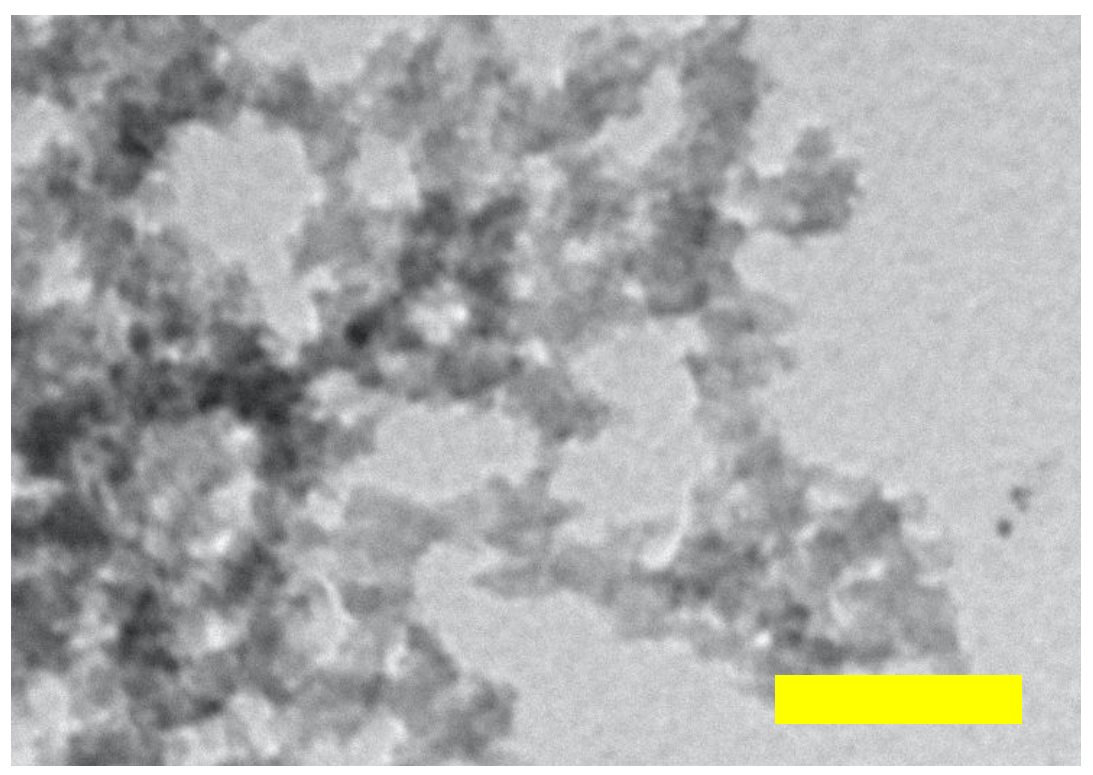

Figure S3. SEM image for raspberry nanoparticles synthesized with the feeding ratio of styrene : trimethyl benzene $=1: 9$. The scale bar represents $100 \mathrm{~nm}$.

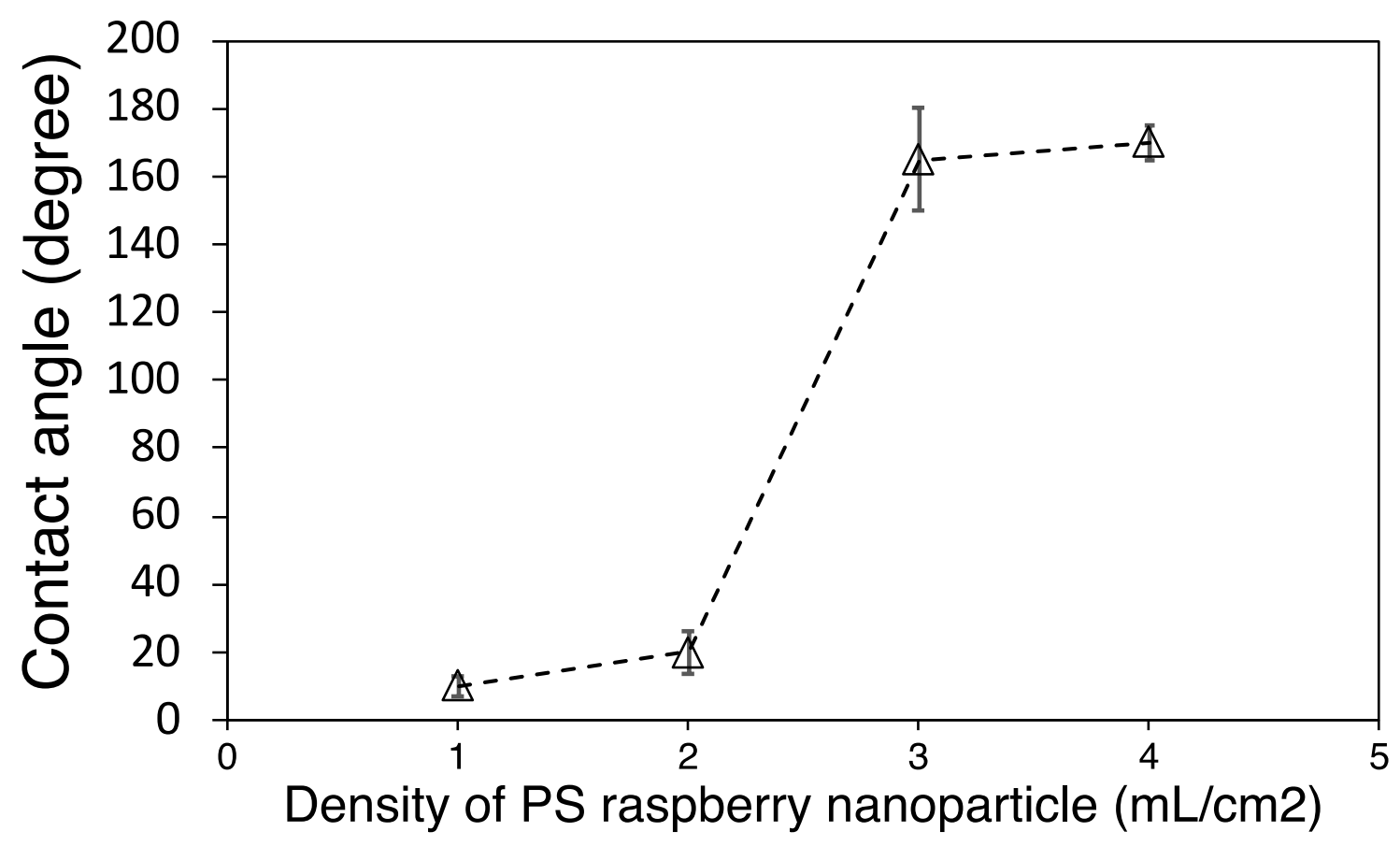

Figure S4. Contact angle of water droplet on PS raspberry nanoparticle coated on the surface in response to the density of nanoparticles on the substrate. 


\section{References}

(1) Van Oss, C. J.; Chaudhury, M. K.; Good, R. J. Advances in Colloid and Interface Science 1987, 28, 35-64.

(2) Zenkin, S.; Kos, Š.; Musil, J. Journal of the American Ceramic Society 2014, 97 (9), 2713-2717.

(3) Zhang, R.; Azimi, G. ACS Appl. Mater. Interfaces 2020.

https://dx.doi.org/10.1021/acsami.0c11353

(4) Van Oss, C. J.; Good, R. J.; Chaudhury, M. K. Journal of Colloid and Interface Science 1986, 111 (2), 378-390.

(5) Apparent and Microscopic Contact Angles, 1st ed.; Mittal, K. L., Drelich, J., Laskowski, J. S., Eds.; CRC Press: London, 2014.

(6) Fundamentals of Adhesion and Interfaces, 1st ed.; Rimai, D. S., DeMejo, L. P., Mittal, K. L., Eds.; VSP: Utrecht, The Netherlands, 1995.

(7) Van Oss, C. J.; Ju, L.; Chaudhury, M. K.; Good, R. J. Journal of Colloid and Interface Science 1989, 128 (2), 313-319.

(8) Polymer Surface Modification: Relevance to Adhesion; Mittal, K. L., Ed.; CRC Press: Boston, 2004; Vol. 3.

(9) Polymer Interfaces and Emulsions; Esumi, K., Ed.; Marcel Dekker, Inc.: New York, 1999.

(10) Adhesion Science and Technology; Mizumachi, H., Ed.; Gordon and Breach Science Publishers: Yokohama, Japan, 1994.

(11) Luner, P. E.; Oh, E. Colloids and Surfaces A: Physicochemical and Engineering Aspects 2001, 181 (1-3), 31-48.

(12) Surface Modification of Polymeric Biomaterials; Ratner, B. D., Castner, D. G., Eds.; Springer US: Boston, MA, 1997.

(13) Coatings of Polymers and Plastics; Ryntz, R. A., Yaneff, P. V., Eds.; Marcel Dekker, Inc.: New York, 2003.

(14) Chae, S.-K.; Lee, C.-H.; Lee, S. H.; Kim, T.-S.; Kang, J. Y. Lab on a Chip 2009, 9 (13), 1957-1961. 